\title{
The Associative-semantic Network for Words and Pictures: Effective Connectivity and Graph Analysis
}

Rik Vandenberghe ${ }^{1,2}$, Yu Wang ${ }^{1}$, Natalie Nelissen ${ }^{1}$, Mathieu Vandenbulcke ${ }^{3}$, Thijs Dhollander ${ }^{4}$, Stefan Sunaert ${ }^{5}$, Patrick Dupont ${ }^{1}$

${ }^{1}$ Laboratory for Cognitive Neurology, Department of neurosciences, KU Leuven;

${ }^{2}$ Neurology Department, University Hospitals Leuven, Belgium;

${ }^{3}$ Psychiatry Department, University Hospitals Leuven, Leuven, Belgium;

${ }^{4}$ Medical Imaging Research Center (MIRC), University of Leuven and University Hospitals Leuven, Belgium;

${ }^{5}$ Radiology Department, University Hospitals Leuven, Belgium

Correspondence to: Rik Vandenberghe, M.D., Ph.D., Neurology Department, University Hospitals Leuven, Herestraat 49, 3000 Leuven, Belgium, e-mail: rik.vandenberghe@uz.kuleuven.be, phone ++32 (0)16 344280, fax: ++ 32 (0)16 3444285

Keywords: semantic, language, lexical, visual word form area, fMRI, effective connectivity

Brain and Language 127 (2013), 264-272

DOI: http://dx.doi.org/10.1016/j.bandl.2012.09.005 


\begin{abstract}
Explicit associative-semantic processing of words and pictures activates a distributed set of brain areas that has been replicated across a wide range of studies. We applied graph analysis to examine the structure of this network. We determined how the left ventral occipitotemporal transition zone (vOT) was connected to word-specific areas. A modularity analysis discerned 4 communities: one corresponded to the classical perisylvian language system, including superior temporal sulcus (STS), middle temporal gyrus (GTm) and pars triangularis of the inferior frontal gyrus (GFi), among other nodes. A second subsystem consisted of vOT and anterior fusiform gyrus along with hippocampus and intraparietal sulcus. The two subsystems were linked through a unique connection between vOT and GTm, which were hubs with a high betweenness centrality compared to STS and GFi which had a high local clustering coefficient. Graph analysis reveals novel insights into the structure of the network for associative-semantic processing.
\end{abstract}

\title{
1 Introduction
}

Until recently, the vast majority of functional imaging data were based on univariate voxelwise comparisons between conditions. The maps of significant differences were often described as 'systems' or 'networks' but this commonly went beyond the actual data analysis. In the current paper we will apply a graph theoretical approach (Bullmore \& Sporns, 2009; Buckner et al., 2009; He \& Evans, 2010; Bullmore \& Bassett, 2011) to characterize the network for associative-semantic processing. As a mathematical technique, graph theory has been known for long. Its application however to imaging data is of a more recent date and has steadily increased in popularity. Graph analysis has been applied to a number of imaging modalities: structural (He et al., 2007, 2008) and resting-state MRI (Nelson et al., 2010), diffusion-tensor imaging (DTI) (Gong et al., 2009) as well as task-related fMRI (Buckner et al., 2009). Compared to dynamic causal modelling (DCM) (Penny et al., 2004), graph analysis does not require the a priori definition of a restricted set of models (nodes, intrinsic feedforward and feedback connections, modulations) by the experimenter. The associative-semantic network consists of a large number of nodes and an even exponentially larger number of possible functional connections. Given the extent of the network and the significant lacunes that remain in our knowledge about its internal connectivity structure, graph analysis was optimally suited for this research purpose.

A highly consistent pattern of activation in functional imaging can be obtained when explicit associative-semantic judgments are compared to lower-level tasks such as visuoperceptual judgments of size for words and pictures (Vandenberghe et al., 1996; Vandenbulcke et al., 2005, 2007; Nelissen et al., 2009, 2011). Regions activated both for words and pictures include the left ventral occipitotemporal transition zone (vOT) (Vandenberghe et al., 1996; Buckner et al., 2000; Van Doren et al., 2010; Seghier \& Price, 2011), left posterior middle temporal gyrus (Chertkow et al., 1997; Vandenbulcke et al., 2007; Whitney et al., 2011), the anterior temporal pole (Hodges et al., 1992; Vandenberghe et al., 1996; Rogers \& McClelland, 2004), left ventral anterior temporal cortex (Jefferies \& Lambon Ralph, 2006; Visser et al., 2012) and left anterior inferior frontal gyrus (Goldberg et al., 2007). Other areas are activated more during semantic processing specifically for words compared to pictures or vice versa. Such areas include the triangular and opercular part of the inferior frontal gyrus, the posterior third of STS for words (Vandenberghe et al., 1996; Vandenbulcke et al., 2007) and the right fusiform gyrus for pictures (Vandenbulcke et al., 2006; Thierry \& Price, 2006). It also contains more domain-general (Binder et al., 2009) regions such as the inferior frontal sulcus (Wagner et al., 1997; Van Doren et al., 2010), 
middle frontal gyrus (Demb et al., 1995; Vandenberghe et al., 1996), and intraparietal sulcus. In this context, we use the term 'domain-general' to refer to cognitive processes that may not be exclusive for tasks involving language or semantic processing and that may be invoked during a wider variety of tasks, e.g. due to their role in selective attention or executive control. A similar distribution of activations has been reported across a wide variety of experiments of semantic processing (Binder et al., 2009). To our knowledge, this set of activations has not been analyzed mathematically at the systems level until now. The first aim of the current study was to apply a graph theoretical approach to this activity pattern to investigate the network structure.

At a more local level, we were mainly interested in how the junction between left ventral occipital and posterior inferior temporal cortex (ventral occipitotemporal transition zone $(\mathrm{vOT}))$ is connected with the perisylvian word-specific system. vOT is activated across a wide variety of language (Cohen et al., 2000, 2002; Jobard et al., 2003; Price \& Devlin, 2003; Dmonet et al., 2005; Vinckier et al., 2007; Seghier \& Price, 2011; Price \& Devlin, 2011; Woodhead et al., 2011; Hellyer et al., 2011) as well as picture processing paradigms (Buckner et al., 2000; Price \& Devlin, 2003; Seghier \& Price, 2011; Price \& Devlin, 2011). It lies at the transition between ventral occipital and ventral temporal cortex and contains the mid-fusiform gyrus (Seghier \& Price, 2011; Price \& Devlin, 2011; Woodhead et al., 2011; Hellyer et al., 2011). It overlaps with the visual word form area (Cohen et al., 2000, 2002; Vinckier et al., 2007). Its y coordinate $(-58 \pm 5 \mathrm{~mm})$ lies slightly posterior to the basal temporal language area $(-41 \pm 6 \mathrm{~mm})$ area, the anterior fusiform gyrus and the inferior temporal gyrus (Jobard et al., 2003; Dmonet et al., 2005). It is neuroanatomically and functionally distinct from the ventral anterior temporal cortex that lies at a distance more anteriorly and has been implicated in multimodal processing of conceptual representations (Visser \& Lambon Ralph, 2011; Visser et al., 2012). Rather than an anatomically or functionally well-delineated area, vOT is a conglomerate of areas fulfilling different functions, with a posterior-anterior gradient (Jobard et al., 2003; Dmonet et al., 2005; Seghier \& Price, 2011).

Many functional interpretations of vOT implicitly assume a connectivity pattern with the language network but this has not been directly tested empirically. For instance, if one considers vOT as an 'entry point' to the word-specific system (Dmonet et al., 2005), one would expect functional connectivity between vOT and language-specific areas such as posterior STS. Likewise, if vOT integrates top-down predictions from the language system with bottom-up visual input (Price \& Devlin, 2011) or reflects the interaction between the 'triangular network' of orthography, phonology and semantics (Woodhead et al., 2011; Hellyer et al., 2011), one would expect a high degree of connectivity with word-specific areas in posterior temporal or inferior frontal cortex. Alternatively, left vOT has also been implicated in more 'generic' functions (Van Doren et al., 2010, 2012). According to one hypothesis, it belongs to a long-distance feedback loop together with the inferior frontal sulcus that mediates visual short-term memory, enhancing perceptual identification, conscious perception and episodic memory encoding (Van Doren et al., 2010, 2012). According to a related hypothesis, based on a meta-analysis of reading studies (Jobard et al., 2003), it is implicated in segmentation and classification of visual stimuli in familiar units. The second aim therefore of the current study was to evaluate how vOT is connected with word-specific areas, such as STS. 


\section{Subjects and Methods}

\section{$2.1 \quad$ Subjects}

Thirty-three healthy elderly subjects, aged between 54 and 89 years of age (19 M/14 F; mean age $67.2 \mathrm{yr}+/-8.5$ years), without neurological or psychiatric history, participated. All were strictly right-handed, free of psychotropic or vasoactive drugs, had a normal structural MRI and scored within the published norms on a standard conventional neuropsychological assessment. This sample has already been published as control group for fMRI studies of language and semantic processing in primary progressive aphasia (Vandenbulcke et al., 2005), stroke (Vandenbulcke et al., 2006), amnestic mild cognitive Impairment (Vandenbulcke et al., 2007) and Alzheimer's disease (Nelissen et al., 2007, 2011).

\subsection{Experimental paradigm}

Stimuli were projected onto a screen $28 \mathrm{~cm}$ in front of the subjects' eyes. The design of the fMRI experiment was factorial (Vandenberghe et al., 1996; Vandenbulcke et al., 2005, 2006; Nelissen et al., 2009). The first factor, task, had two levels: Associative-semantic versus visuoperceptual judgment. The second factor, input modality, also had two levels: Pictures versus printed words. The associative-semantic condition consisted of a modified version of the Pyramids and Palm Trees test (Hodges et al., 1992). During a trial, a triplet of stimuli was presented for $5250 \mathrm{~ms}$, one stimulus on top (the sample stimulus) and one in each lower quadrant (the test stimuli). Subjects had to press a left- or righthand key depending on which of the two test stimuli matched the sample stimulus more closely in meaning. A given triplet was presented in either the picture or the word format and this was counterbalanced across subjects. In the visuoperceptual control condition, a stimulus was presented in three different sizes. Subjects had to press a left or right-hand key depending on which of the two test stimuli matched the sample stimulus more closely in size on the screen. Two successive trials were separated by a $1500 \mathrm{~ms}$ interval. Each epoch, i.e. a block of trials of the same type, consisted of 4 trials (total duration $27 \mathrm{~s}$ ).

Stimuli were presented at $3.8 \mathrm{deg}$ eccentricity. The pictures were taken from the Snodgrass-Vanderwart set. Mean picture size was $5.6 \mathrm{deg}$ and letter size $1.5 \mathrm{deg}$.

\subsection{Image acquisition}

A 1.5 tesla Siemens Sonata system (Siemens Medical Solutions, Erlangen, Germany) equipped with an 8-channel receive-only head coil (MRI Devices Corp., Waukesha, USA) provided a T1-weighted structural volume (coronal inversion recovery prepared threedimensional gradient echo images; Inversion time $800 \mathrm{~ms}$, TE/TR 3.93/1950 ms) as well as T2* echo-planar images (EPI) (42 sagittal slices; voxel size $3.6 \times 3 \times 3 \mathrm{~mm}^{3}$; TE/TR 40/3000 ms). Usage of the GeneRalized Autocalibrating Partially Parallel Acquisitions (GRAPPA) method (Griswold et al., 2002) maximized sensitivity for anterior temporal activity changes and minimized susceptibility artefacts. Scans were acquired between 2003 and 2007 (Vandenbulcke et al., 2005, 2006, 2007; Nelissen et al., 2007), prior to the development of Spin-Echo EPI as a means to reduce anterior temporal susceptibility artifact (Visser \& Lambon Ralph, 2011; Visser et al., 2012). A total of 108 volumes were acquired during each run. Each run consisted of 3 replications of each of the 4 conditions. Subjects underwent 4 to 6 runs each. 


\subsection{Image preprocessing}

We used the normalized and smoothed data from previous studies (Vandenbulcke et al., 2005, 2006, 2007; Nelissen et al., 2007) which were spatially transformed using Statistical Parametric Mapping (SPM2; Wellcome Department of Cognitive Neurology, London, UK; http://www.fil.ion.ucl.ac.uk/spm). In brief, EPI images were realigned and the anatomical MRI was coregistered to the mean realigned EPI image, the coregistered MRI was normalized to a custom-made T1 template of elderly subjects (Nelissen et al., 2007) in Montreal Neurological Institute (MNI) space, and the normalization matrix was subsequently applied to all individual EPI images. These images (voxel size of $3 \times 3 \times 3 \mathrm{~mm}^{3}$ ) were smoothed with a Gaussian kernel $\left(6 \times 6 \times 6 \mathrm{~mm}^{3}\right)$. Using SPM8, we applied high-pass filtering $(\mathrm{FWHM}=270 \mathrm{~s}$ ) and low-pass filtering consisting of a canonical hemodynamic response function (hrf) to the data. The epoch related response was modeled by a canonical hrf convolved with a boxcar.

\subsection{Network nodes and time series}

A random effects analysis was performed for the main effect of task (associative semantic judgment $>$ visuoperceptual judgment). We used a statistical threshold at the voxel level of uncorrected $P<0.001$ combined with a family wise error (FWE) corrected $p<$ 0.05 at the cluster level. Suprathreshold clusters were ordered according to decreasing size and within each cluster, all suprathreshold local maxima were ordered according to decreasing t value. Next, we selected the MNI coordinates of local maxima which were located at least $20 \mathrm{~mm}$ apart from each other, starting from the coordinates of the first local maximum in our ordered list. In each coordinate, we defined a sphere with a radius of $6 \mathrm{~mm}$ and intersected this sphere with the underlying cluster. This gave us a set of voxels which constitute the volume of interest (VOI) of the network node around that coordinate. Note that for all subjects the same VOIs derived from the group analysis were used. Alternative approaches to define the nodes are to start from VOIs based on a priori atlases (He et al., 2007, 2008) or to include each gray matter voxel (Buckner et al., 2009).

To assess the between-subject variability of the nodes, we calculated for each node the percentage subjects for which a voxel with an uncorrected $P<0.05$ was found within a distance of $6 \mathrm{~mm}$ from the group local maximum. We also calculated the contribution coefficient (Seghier et al., 2007). If every subject contributed equally to the group spm T map, the contribution coefficient equals - in our case - 1/33.

In each voxel of the VOI, we extracted the time series after whitening, filtering and removing effects of no interest (session specific effects) using SPM8 code. Finally, the average time series in the network node was calculated as the mean of the time series in the voxels belonging to the corresponding VOI. Time series of the different runs were concatenated.

\subsection{Partial correlation coefficients}

The connectivity between nodes was determined by the partial correlations between the network nodes, quantifying the unique relationship between each pair of nodes. The calculation of the partial correlations was based on calculating the inverse of the covariance matrix. After applying Fisher's r-to-Z transformation to each partial correlation a random effects analysis was performed to identify those connections which were significantly different from zero at the group level. The significance level was set at $p<0.05$ Bonferroni 
corrected for the total number of possible connections. Significant partial correlations are closely related to effective connectivity (Marrelec et al., 2006, 2009).

\subsection{Graph analysis}

Graph analysis was performed using the brain connectivity toolbox

(https://sites.google.com/a/brain-connectivity-toolbox.net/bct/Home (Rubinov \& Sporns, 2010)).

Prior to graph analysis, we binarized the connections based on the statistical significance (He et al., 2007) of the partial correlation coefficients. The statistical threshold was set to $P<0.05$ corrected for the number of possible connections. This threshold determines the 'density' of the network, i.e. the number of supra-threshold connections over the total number of possible connections.

We calculated two global network measures: the characteristic path length of the graph and the mean clustering coefficient. The path length of node $i$ is the average distance to each other node excluding isolated nodes. The distance between two nodes is the shortest path length between these nodes. The characteristic path length of the graph is the path length averaged over all nodes. The mean clustering coefficient of the graph is the clustering coefficient averaged over all nodes. The clustering coefficient of node $i$ is the fraction of neighbors of node $i$ that are neighbors of each other. We will also report the normalized values of the characteristic path length and the mean clustering coefficient, i.e. the values divided by the value obtained in an equivalent random network. A normalized mean clustering coefficient $>1$ and a normalized characteristic path length of approximately 1 are commonly considered to indicate small-world characteristics of the network.

To evaluate the robustness of our graph analysis, we examined the impact of sample size on the global graph measures. We calculated the difference between the global graph measures as a function of the number of subjects included, compared to using the full sample $(\mathrm{n}=33)$. We randomly selected a subset of cases. The relative error was the average of the relative error over all realizations of this random sample (with a maximum of 500 realizations) (Fig. 2).

Apart from path lenght and local clustering coefficient, typical graph measures at the local level are also degree and betweenness centrality. The degree of node $i$ is the number of edges (connections) linked to node $i$. The betweenness centrality of node $i$ is the fraction of shortest paths within the graph that pass through node $i$. Betweenness centrality is a measure of how pivotal a given node is in the overall network. Hubs are typically characterized by a high degree, a high betweenness centrality, a short average path length and a low clustering coefficient (Sporns et al., 2007; Bullmore \& Sporns, 2009). To identify such hubs, we calculated a hub score. The hub score is the sum of the dummy values for 4 criteria (each set at 1 or 0 depending on whether or not the criterion is fulfilled, with a maximum of 4) (van den Heuvel et al., 2010; Heitger et al., 2012). These criteria are whether the node belongs to the top $20 \%$ of nodes

1. showing the highest degree

2. showing the lowest path length

3. showing the lowest local cluster coefficient

4. showing the highest betweenness centrality. 
When a node had a hub score of 2 or more, it was marked as a hub (van den Heuvel et al., 2010). The hub scores will be provided for each node in Table 1.

Graph analysis also allows one to detect of which 'modules' or 'communities' a network is built. Communities are non-overlapping groups of nodes with a high number of withincommunity connections and a low number of between-community connections. We used the algorithm of Newman (Newman, 2006a,b) as implemented in the Brain Connectivity Toolbox to determine the community structure of the network. In this modularity analysis, the algorithm starts from a random order of the nodes. Across different iterations (realizations) of the analysis this order is randomized, in our instance for a total of 100 realizations. We evaluated how systematically the same community structure recurred between these different realisations of the community structure. If two nodes were assigned to a same community structure in $80 \%$ of these realizations, the assignment was considered relatively stable. Nodes that were assigned to a same community across $95 \%$ or more of realizations, will be considered most robust and be the main focus of this report.

As a further means to determine the robustness of our modularity analysis, we also examined the impact of network density on the community structure. One of the factors determining network density is the threshold applied when the connections are binarized. Network density therefore can be manipulated by varying the statistical threshold for binarizing the connections. We varied this threshold between 3 different possibilities: $P<0.1, P<0.05$ and $P<0.01$. All $P$ values are corrected for the number of possible connections.

The network will be visualized using Pajek (http://pajek.imfm.si) and a KamadaKawai energy layout (Kamada \& Kawai, 1989; Batagelj \& A.Mrvar, 1998). In this approach, two nodes are considered as connected by a spring with a length equal to the strength of the partial correlation. The final layout is then given by minimization of the energy of the layout.

\section{Results}

\subsection{Nodes}

The thresholded $t$ map of the main effect of task is shown in figure 1A. The median size of the intersection of the sphere (with radius $6 \mathrm{~mm}$ around the center coordinate) and the thresholded group spmT map was 29 voxels (range 3 - 33 voxels). The number of subjects with at least one voxel with an uncorrected $p<0.05$ (for the main effect of task) within the sphere was $88 \%$ (median value; range $58-100 \%$ ). The contribution coefficient of every subject is given in figure $1 \mathrm{~B}$.

\subsection{Global graph measures}

The mean clustering coefficient of the associative-semantic network was 0.32 and the characteristic path length 3.17. The corresponding values for an equivalent random network (with 57 nodes and 124 connections) using 1000 randomizations were 0.07 and 2.84, respectively. The normalized values therefore for mean clustering coefficient and characteristic path length were 4.57 and 1.12, respectively. The network therefore exhibits small-world characteristics.

To evaluate the impact of sample size on these estimates, we calculated the error of these global graph measures as function of sample size, relative to using the full sample 

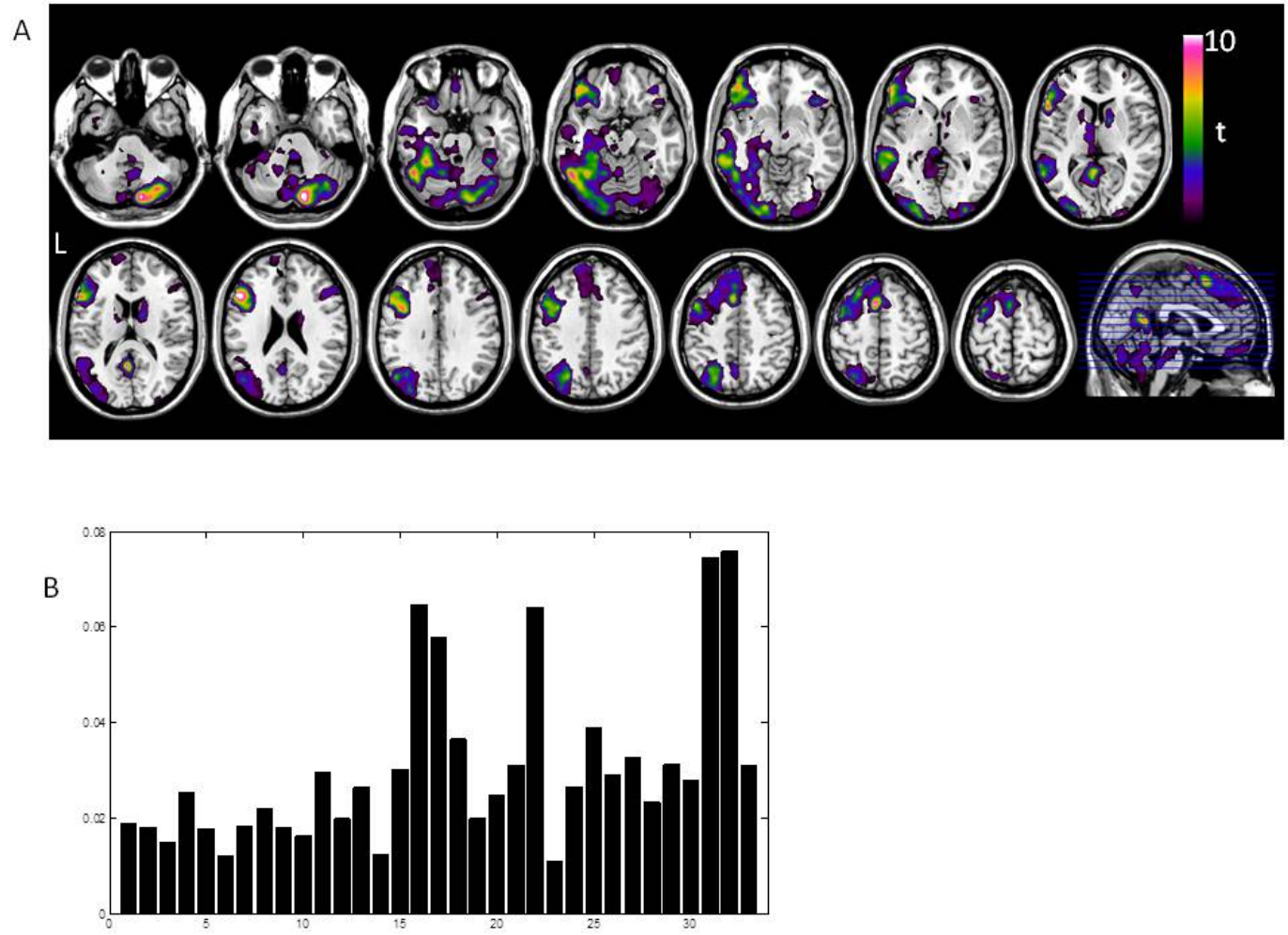

Figure 1: A. spmT map on transverse sections, corresponding to the contrast of associative-semantic versus visuoperceptual judgment, thresholded at voxel-level uncorrected $P<0.001$ and cluster-level FWE corrected $P<0.05$. B. Contribution coefficient for each of the 33 subjects (Seghier et al., 2007). 


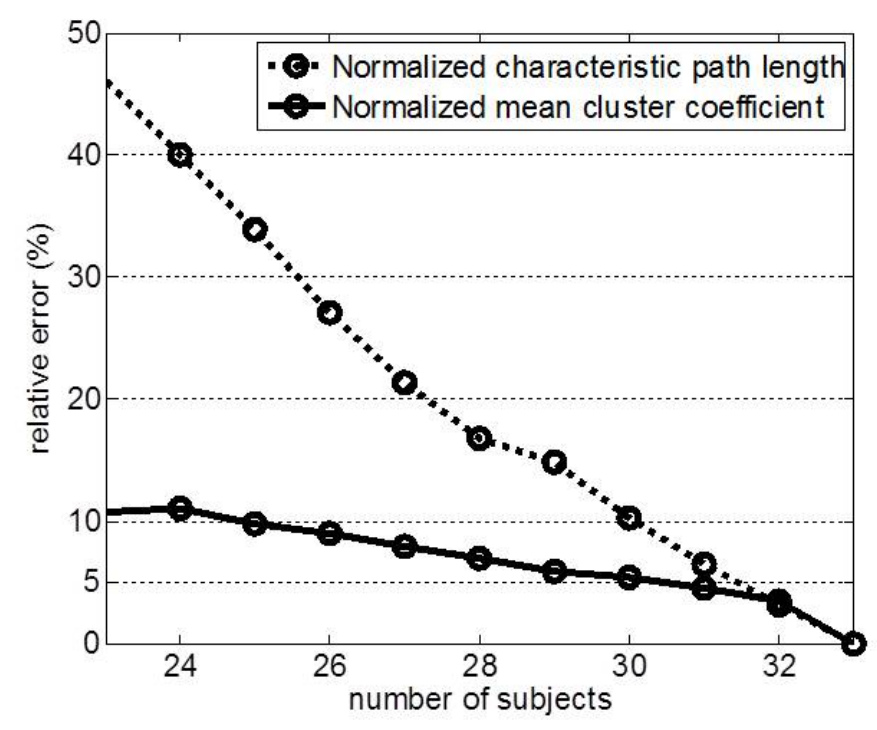

Figure 2: Relative error as a function of sample size. The relative error is the difference between the observed value and the value obtained in the full dataset $(\mathrm{n}=33)$ divided by the value of the full dataset. $\mathrm{X}$ axis: sample size. $\mathrm{Y}$ axis: relative error in $\%$.

$(\mathrm{n}=33)$. Error linearly decreased as a function of sample size, with values of $10 \%$ for 31 or more subjects (Fig. 2).

\subsection{Local graph measures and community structure}

The final network is shown in Fig. 3. The local network measures (degree, path length, local clustering coefficient, betweenness centrality and hub score) are given in Table 1 for nodes with a degree $>3$.

Analysis of the community structure revealed 6 subsystems (Figure 3, table 1). The composition of 4 of these subsystems was highly robust: it was present in $95 \%$ of realisations (Table1, symbol + ). These communities were also robust when we manipulated the network density by varying the statistical threshold either to a corrected $P<0.1$ (density $9.1 \%$ ) or $P<0.01$ (density $6.3 \%$ ) instead of $P<0.05$ (density of $7.8 \%$ ) (table 1 , symbol $*)$. We will mainly focus on these 4 most robust communities.

Three were nearly exclusively left-hemispheric, the fourth was mainly right-sided and located more posteriorly. Among the left-sided communities, one was mainly perisylvian, the second community extended from IPS to vOT extending ventromedially to the parahippocampal gyrus and the hippocampus, and the third covered the dorsolateral prefrontal and medial prefrontal cortex. Its most inferior component was the inferior frontal junction.

The perisylvian community (Figure 3, red dots) consisted of posterior STS and anterior STS, middle temporal gyrus and the anterior temporal pole, pars orbitalis of the frontal operculum and the anterior inferior frontal gyrus, as well as the left angular and left supramarginal gyrus. The left angular gyrus and the left posterior middle temporal gyrus 


\begin{tabular}{|c|c|c|c|c|c|c|c|c|c|}
\hline $\mathrm{x}$ & $\mathrm{y}$ & $\mathrm{z}$ & $\begin{array}{l}\text { anatomical } \\
\text { location }\end{array}$ & degree & $\begin{array}{c}\text { path } \\
\text { length }\end{array}$ & $\begin{array}{l}\text { clustering } \\
\text { coefficient }\end{array}$ & $\begin{array}{c}\text { betweenness } \\
\text { centrality }\end{array}$ & $\begin{array}{l}\text { hub } \\
\text { score }\end{array}$ & community \\
\hline-48 & -69 & 30 & L angular gyrus & 8 & 2.6 & 0.14 & 0.12 & 4 & red ${ }^{*}+$ \\
\hline-63 & -39 & -6 & L posterior GTm & 7 & 2.7 & 0.33 & 0.10 & 2 & red $*+$ \\
\hline-60 & -54 & 9 & L posterior STS & 6 & 3.1 & 0.47 & 0.03 & 0 & red $*+$ \\
\hline-51 & 21 & 24 & L GFi pars triangularis & 5 & 3.0 & 0.40 & 0.03 & 0 & red + \\
\hline-60 & -45 & 27 & L supramarginal gyrus & 5 & 3.0 & 0.30 & 0.03 & 0 & red *+ \\
\hline-54 & 27 & -3 & L GFi pars orbitalis & 5 & 3.2 & 0.50 & 0.01 & 0 & red + \\
\hline-57 & -12 & -9 & L anterior STS & 4 & 3.5 & 0.50 & 0.01 & 0 & red *+ \\
\hline-39 & 39 & -18 & $\mathrm{~L}$ anterior $\mathrm{GFi}$ & 4 & 3.1 & 0.67 & 0.00 & 0 & red + \\
\hline-30 & -66 & 60 & L intraparietal sulcus (IPS) & 6 & 2.6 & 0.40 & 0.11 & 2 & yellow *+ \\
\hline-54 & -57 & -18 & L vOT & 5 & 2.6 & 0.20 & 0.11 & 2 & yellow $*+$ \\
\hline-39 & -87 & 0 & L middle occipital gyrus (GOm) & 8 & 2.5 & 0.29 & 0.10 & 3 & yellow $*+$ \\
\hline-15 & -93 & -9 & L lingual gyrus & 7 & 2.5 & 0.19 & 0.10 & 3 & yellow $*+$ \\
\hline-36 & -42 & -24 & $\mathrm{~L}$ anterior fusiform & 8 & 2.5 & 0.25 & 0.08 & 3 & yellow $*+$ \\
\hline-24 & -66 & 39 & L intraparietal sulcus (IPS) & 6 & 2.6 & 0.40 & 0.04 & 0 & yellow $*+$ \\
\hline-30 & -33 & -6 & L hippocampus & 4 & 3.1 & 0.33 & 0.02 & 0 & yellow $*+$ \\
\hline 33 & -39 & -24 & $\mathrm{R}$ anterior fusiform & 6 & 3.6 & 0.33 & 0.04 & 0 & green *+ \\
\hline 27 & -96 & -3 & $\mathrm{R}$ inferior occipital gyrus & 5 & 3.1 & 0.40 & 0.03 & 0 & green $*+$ \\
\hline 42 & -78 & -9 & $\mathrm{R}$ inferior occipital gyrus & 5 & 3.1 & 0.40 & 0.02 & 0 & green $*+$ \\
\hline 30 & -69 & -54 & $\mathrm{R}$ cerebellum & 5 & 3.6 & 0.30 & 0.02 & 0 & green + \\
\hline 30 & -93 & 18 & R middle occipital gyrus (GOm) & 4 & 3.1 & 0.67 & 0.01 & 0 & green $*+$ \\
\hline-39 & 6 & 33 & $\mathrm{~L}$ inferior frontal junction (IFJ) & 7 & 2.8 & 0.19 & 0.10 & 2 & blue *+ \\
\hline-3 & 15 & 54 & dorsomedial prefrontal & 8 & 2.6 & 0.25 & 0.09 & 3 & blue *+ \\
\hline-24 & 24 & 54 & L superior frontal sulcus (SFS) & 7 & 2.8 & 0.29 & 0.04 & 1 & blue *+ \\
\hline-9 & 54 & 45 & L superior frontal gyrus (GFs) & 6 & 2.9 & 0.47 & 0.04 & 0 & blue $*+$ \\
\hline-33 & 6 & 60 & L superior frontal gyrus (GFs) & 5 & 2.8 & 0.40 & 0.03 & 0 & blue $*+$ \\
\hline-9 & 30 & 33 & $\mathrm{~L}$ anterior cingulate & 6 & 3.0 & 0.40 & 0.02 & 0 & blue $*+$ \\
\hline-9 & 36 & 60 & L superior frontal gyrus (GFs) & 6 & 3.1 & 0.47 & 0.02 & 0 & blue *+ \\
\hline-3 & -57 & 15 & precuneus & 6 & 2.6 & 0.33 & 0.07 & 1 & purple $^{*}+$ \\
\hline-12 & -3 & 9 & $\mathrm{~L}$ anterior thalamus & 6 & 2.9 & 0.33 & 0.03 & 0 & purple \\
\hline 12 & 3 & 12 & $R$ caudate & 4 & 3.1 & 0.50 & 0.01 & 0 & purple + \\
\hline-3 & -33 & 3 & posterior thalamus & 7 & 2.6 & 0.29 & 0.08 & 3 & cyan \\
\hline-15 & -54 & -18 & L cerebellum & 6 & 2.6 & 0.07 & 0.08 & 2 & cyan + \\
\hline 45 & 21 & 27 & R GFi pars triangularis & 4 & 2.6 & 0.00 & 0.08 & 3 & cyan \\
\hline-21 & -33 & -51 & L cerebellum & 4 & 3.0 & 0.00 & 0.06 & 1 & cyan + \\
\hline-3 & -63 & 45 & precuneus & 6 & 2.7 & 0.27 & 0.05 & 0 & cyan \\
\hline 6 & -54 & -42 & vermis & 4 & 3.1 & 0.00 & 0.03 & 1 & cyan + \\
\hline
\end{tabular}

Table 1: Ranking of nodes per community according to betweenness centrality and, next, degree. Only local graph measures of nodes with a degree $>3$ are shown. $\mathrm{x}, \mathrm{y}, \mathrm{z}$ are the MNI coordinates of the center of the nodes; degree is the degree of the node, path length is the average shortest path length of the node to any other node (excluding 3 nodes which were isolated in the graph), community refers to the colour coding used in figure 3. Hubs (with a hub score of 2 or more) are indicated in bold. ${ }^{*}$ denotes robustness of community structure when varying the density of the network. Nodes marked with '+' are assigned to a given community in $95 \%$ of the realizations. Abbreviations: vOTC: ventral occipitotemporal transition zone; GTm: middle temporal gyrus; STS: superior temporal sulcus; GFi: inferior frontal gyrus; L: left.; R: right. 


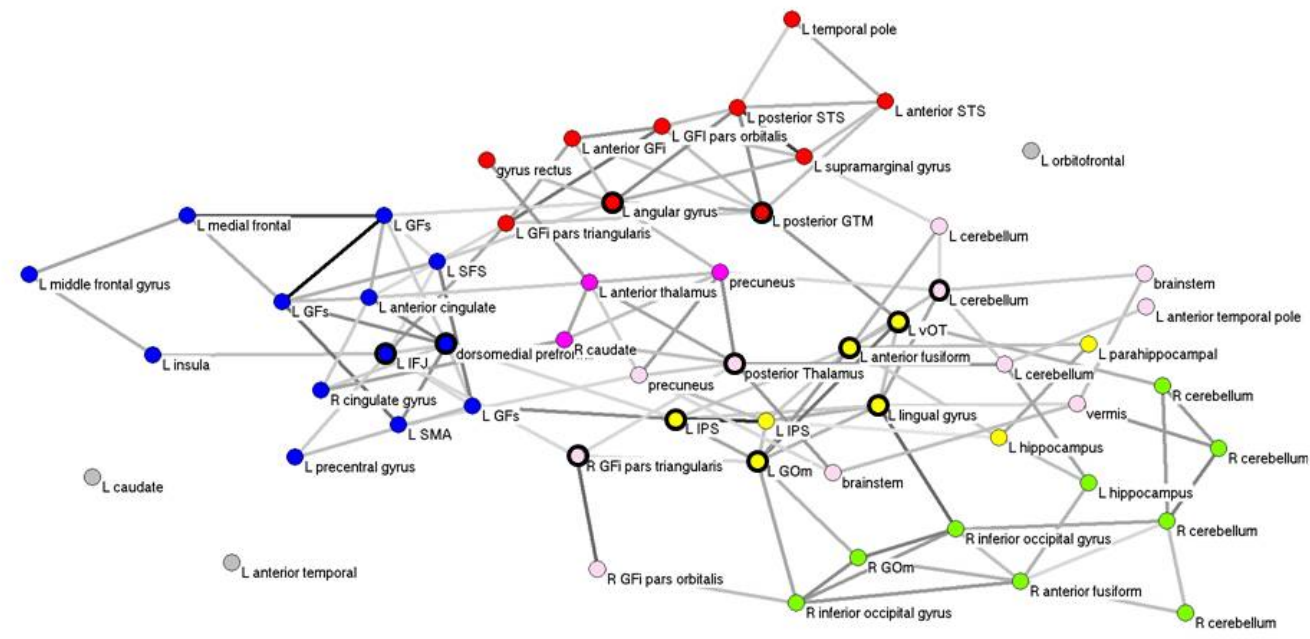

Figure 3: Graph of the associative-semantic network. The connection strength is determined by the partial correlation. Only connections which are significant at corrected $p<0.05$ are shown. Nodes belonging to a same 'community' are given a same color. The two communities most pertinent to the current purposes are: (red) the left perisylvian module including posterior and anterior STS, posterior GTm, the anterior temporal pole, angular and supramarginal gyrus, GFi (anterior, pars orbitalis and pars triangularis), gyrus rectus and the orbitofrontal gyrus and (yellow) the left ventral occipitotemporal module including vOT, IPS, anterior fusiform, lingual and parahippocampal gyrus, GOm, hippocampus and caudate). The 4 additional communities are colored in blue, purple, green and cyan, respectively. Nodes encircled with a black border are considered as hubs in the network. 


\begin{tabular}{lrrrrc} 
& $\mathrm{x}$ & $\mathrm{y}$ & $\mathrm{z}$ & $\begin{array}{c}\text { corrected } \\
\text { p-value }\end{array}$ & $\begin{array}{c}\text { partial } \\
\text { correlation }\end{array}$ \\
\hline A. Cortical connectivity of left vOT $(-54,-57,-18)$ \\
L middle occipital gyrus (GOm) & -39 & -87 & 0 & $8.9 \mathrm{E}-05$ & 0.13 \\
L posterior GTm & -63 & -39 & -6 & $4.8 \mathrm{E}-02$ & 0.10 \\
L anterior fusiform & -36 & -42 & -24 & $1.2 \mathrm{E}-03$ & 0.07 \\
R cerebellum & 36 & -69 & -30 & $5.4 \mathrm{E}-04$ & 0.07 \\
L lingual gyrus & -15 & -93 & -9 & $3.6 \mathrm{E}-03$ & 0.06 \\
\hline B. Cortical connectivity of left posterior STS & $(-60,-54,9)$ & \\
L supramarginal gyrus & -60 & -45 & 27 & $4.4 \mathrm{E}-10$ & 0.19 \\
L angular gyrus & -48 & -69 & 30 & $9.1 \mathrm{E}-08$ & 0.12 \\
L posterior GTm & -63 & -39 & -6 & $6.9 \mathrm{E}-05$ & 0.11 \\
L anterior STS & -57 & -12 & -9 & $7.0 \mathrm{E}-05$ & 0.08 \\
L GFi pars orbitalis & -54 & 27 & -3 & $5.3 \mathrm{E}-03$ & 0.06 \\
L anterior temporal pole & -54 & 12 & -21 & $7.4 \mathrm{E}-03$ & 0.05 \\
\hline
\end{tabular}

Table 2: Cortical nodes with a significant partial correlation with left vOT (A) and left posterior STS (B), respectively, ranked based on the strenght of the partial correlation coefficient. Abbreviations: vOTC: ventral occipitotemporal transition zone; GTm: middle temporal gyrus; STS: superior temporal sulcus; GFi: inferior frontal gyrus; L: left.; R: right.

were the hubs of this network (Figure 3 red, Table 1 ).

Left vOT belonged to a different subsystem together with left anterior fusiform gyrus, left middle occipital gyrus (GOm), left intraparietal sulcus (IPS) and ventromedially the left parahippocampal gyrus and hippocampus (Figure 3, yellow dots). This system clearly lay at a distance from the sylvian fissure, more or less concentrically to the perisylvian community. This module contained a total of 5 hubs: IPS, vOT, GOm, lingual gyrus and the anterior fusiform gyrus (Figure 3 yellow, Table 1).

A third system (Figure 3, blue dots) consisted almost entirely of foci in middle and superior frontal gyrus, both lateral and medial wall as well as cingulate. Two hubs were located in this module: one in the left inferior frontal junction (IFJ) and one in the dorsomedial prefrontal cortex (Figure 3 yellow, Table 1).

Graph analysis also extracted a fourth, mainly right-sided community. It contained the right mid- and anterior fusiform gyrus (Vandenbulcke et al., 2006), as well as the right middle occipital gyrus (Figure 3, green dots). One of the nodes was located in the left hemisphere, i.e. hippocampus.

\subsection{Connectivity between vOT and STS}

Given our primary research question regarding the connectivity between vOT and STS, we also provide more detail about the partial correlations of left vOT $(-54,-57,-18)$ and left posterior STS $(-60,-54,9)$ (Table $2 \mathrm{~A}$ and B, respectively): Both nodes had a relatively strong connection with left posterior GTm but no direct connections with each other. Except for the posterior GTm, no other overlap in neighbours was found between posterior STS and vOT (Table 2). This strongly suggests that vOT and STS are connected with each other through their mutual connection with posterior GTm. The striking difference in connectivity pattern also suggests that they may belong to different communities (Figure 3, yellow versus red dots). 


\section{Discussion}

Graph analysis allowed us to mathematically decompose the distributed network activated by the contrast of associative-semantic with visuoperceptual processing (Vandenberghe et al., 1996; Vandenbulcke et al., 2005, 2006; Nelissen et al., 2009, 2011) into subsystems, or in graph terminology, 'communities'. In a strictly data-driven manner, our modularity analysis dissected the perisylvian network out of the distributed set of activations with remarkable precision. Left temporal and occipital activations fell into two separate communities: this perisylvian subsystem containing STS, pars triangularis, and GTm along with angular gyrus and a second, more or less concentric subsystem extending from IPS to vOT and ventromedially towards the parahippocampal gyrus and the hippocampus. The interface between these two communities consisted of a unique direct connection of vOT with the posterior third of the middle temporal gyrus (Table 2).

All nodes were defined on the basis of a same contrast, preventing any bias that could be induced in our connectivity analysis by differences in contrasts used to define nodes. Neither can proximity between nodes explain the connectivity pattern: To define nodes, we applied a criterion of minimally $2 \mathrm{~cm}$ Euclidean distance and, furthermore, many connections that reached significance bridged relatively large distances, e.g. left posterior STS to left pars orbitalis GFi (Table 2).

The data were acquired prior to the advent of Spin-Echo EPI which substantially increases sensitivity in anterior temporal cortex (Visser et al., 2010; Visser \& Lambon Ralph, 2011). This may explain why we did not obtain activation in the ventral anterior temporal cortex (Visser et al., 2010; Visser \& Lambon Ralph, 2011). The most anterior temporal region we reliably imaged was at $\mathrm{y}=-12$ (anterior STS). More anterior temporal activity foci were rather scarce and usually had a low degree, probably for reasons of fMRI susceptibility. Our data therefore do not allow for any conclusions regarding the position of the anterior temporal regions in the network. This is a shortcoming as some of these anterior temporal nodes may function as hubs (Lambon Ralph et al., 2010).

Among the distributed set of activations (Fig. 1) our modularity analysis dissected out the perisylvian network (Mesulam, 1998, 2008) with remarkable accuracy (Fig. 3). Two of the nodes belonging to the perisylvian community, the posterior middle temporal gyrus and the left angular gyrus were assigned a hub status. Most of the other nodes of this module had a relatively high local clustering coefficient (Table 1). The posterior middle temporal gyrus and the angular gyrus have been found in numerous studies of semantic processing (for a review see (Binder et al., 2009)) and have been previously described as network hubs (Buckner et al., 2009). Both have been implicated in multimodal integration and semantic access (Jobard et al., 2003). Their high betweenness centrality may fit with the hypothesis that they play a role in 'semantic executive control' (Whitney et al., 2012), a process similar to what has been previously denoted as 'semantic access' (Lauro-Grotto et al., 1997). If a region plays a role in semantic control, one would predict a high betweenness centrality since semantic control requires coordination of and interaction with regions that are widely distributed over the cortical surface (Jefferies \& Lambon Ralph, 2006; Noonan et al., 2010; Corbett et al., 2009, 2011). GTm is activated mainly in tasks that require explicit responses related to the identity or the semantic category of the stimuli (Van Doren et al., 2010). The effect of TMS on posterior GTm depends on task demands, in terms of degree of strategic control required during semantic knowledge retrieval (Whitney et al., 2012). The similarity between TMS effects in posterior GTm and GFi fit with the observation that both nodes belonged to the same subsystem (Fig. 3, Table 1). In contrast, TMS of IPS using the same paradigm yields qualitatively different effects, with a domain-independent selection deficit (Whitney et al., 2012). This is con- 
sistent with our observation that IPS belongs to a separate subsystem involved in more generic processes (Fig 3, table 1). The distinction between the perisylvian subsystem and a more general-purpose subsystem including vOT and IPS, among other nodes, is also in agreement with fMRI data directly contrasting orienting based on spatial versus semantic cues. Semantic cueing specifically activates the areas which our graph analysis assigned to the perisylvian subsystem, such as the posterior STS, posterior GTm, GFi and angular gyrus (Cristescu et al., 2006). In contrast, IPS was generally involved in both spatial and semantic cueing (Cristescu et al., 2006), in accordance with its assignment to a more general-purpose community in our graph analysis (Fig. 3).

The angular gyrus had the highest betweenness centrality of all nodes tested. In patients with aphasic stroke in the middle cerebral artery territory, semantic control is impaired and the angular gyrus is one of the areas that is regularly damaged under such conditions (Jefferies \& Lambon Ralph, 2006; Noonan et al., 2010; Corbett et al., $2009,2011)$. Other communities outside the perisylvian subsystem, such as the prefrontal subsystem, may also be involved in executive control, but the angular gyrus differed from these control regions as it was immersed in the perisylvian community according to our graph analysis. This may indicate that its role in regulatory control is specifically related to language and semantic processing, at least under the current experimental paradigm. Its function has been interpreted in terms of multimodal integration and it has been implicated in semantic access (Jobard et al., 2003). The perisylvian subsystem also contained a second inferior parietal node in the supramarginal gyrus. This node corresponded with $50 \%$ probability with human cytoarchitectonic area PF (Caspers et al., 2006, 2008), an area that has been implicated in dictation and repetition, as well as gesture discrimination and imitation (Nelissen et al., 2010).

The perisylvian community also contained several nodes with a high local clustering coefficient but a relatively low betweenness centrality. The local clustering coefficient of a given node $x$ reflects how frequently the neighbours of node $x$ are connected also directly with each other. Using a social-life metaphore, it constitutes a measure of 'cliquishness'. Apart from the left anterior inferior frontal gyrus (Goldberg et al., 2007), the nodes with the highest local clustering coefficients have been typically implicated in word-specific processing, such as the pars orbitalis of the frontal operculum, and left anterior and posterior STS (all above 0.45, table 1). The posterior third of the left STS showed a high local clustering coefficient (0.47) with relatively low betweenness centrality (0.03), which clearly differed from the posterior middle temporal gyrus (0.33 and 0.10 , respectively). Its connectivity profile was also clearly different (Fig.3, Table 2B). These observations confirm the functional dissociation between these 2 juxtaposed posterior temporal regions (Vandenbulcke et al., 2007). Left posterior STS was principally connected with nodes within the perisylvian subsystem, including the angular and supramarginal gyrus, anterior STS, anterior temporal pole, the orbital part of the frontal operculum and posterior GTm. Left posterior STS hypoactivity is associated with slowing of visual word identification speed as well as naming deficits, suggestive of a role of posterior STS in lexical-semantic retrieval (Vandenbulcke et al., 2007). Alternatively, the association between STS activity and word identification speed could also be explained by a role of posterior STS in graphophonological conversion (Jobard et al., 2003). Activity level in left posterior STS also increases with increasing rates of hearing or retrieving words and in noun and verb generation versus rest, which may indicate a role in transient representation of phonological sequences (Wise et al., 2001). From an anatomical perspective, fascicles that form the structural basis for the dense interconnectedness within the perisylvian system are probably the arcuate fascicle, the middle longitudinal fascicle and the extreme capsule (Schmahmann \& Pandya, 2006). 
A second highly robust system was located distally and concentrically to the perisylvian module. vOT was part of this subsystem rather than the perisylvian subsystem. This has implications for the function attributed to vOT. If vOT were an area of interaction between a triangular network of orthography, phonology, and semantics (Woodhead et al., 2011; Price \& Devlin, 2011), one would rather expect it to belong to the perisylvian language subsystem containing the lateral temporal, inferior frontal and inferior parietal nodes. This was not the case. Instead, vOT belonged to a subsystem that also contained IPS, typically implicated in attentional selection (Vandenberghe et al., 2005; Molenberghs et al., 2008; Vandenberghe \& Gillebert, 2009) and hippocampus, typically implicated in episodic memory. In our interpretation, the contribution of this ventral occipitotemporal system relates more to conscious perception, visuoperceptual identification, attentional selection, access of perceptual units to visual short-term memory, and long-term memory (Bundesen et al., 2005). Identification ('categorization') of perceptual units is determined by sensory evidence, relevance and expectancy which determine access of the perceptual units to visual short-term memory (Bundesen \& Habekost, 2008). The interaction account of vOT (Price \& Devlin, 2011) with prediction error is closely related to this proposal. According to this hypothesis, vOT integrates top-down predictions from the language system with bottom-up visual input (Price \& Devlin, 2011). Based on a meta-analysis of reading studies, Jobard et al. (2003) implicated vOT in segmentation and classification of visual stimuli in familiar units. At the moment we do not attribute a specific semantic role to this community but rather a general-purpose function that is not necessarily exclusively related to language or semantic processing. We propose that this system functions as an 'amplifier' of inner representations of perceptual units, mediating conscious perception and enhancing access of these units to other cognitive systems, e.g. involved in episodic memory (Van Doren et al., 2010, 2012). This hypothesis is also compatible with the observation that this community contains a remarkably high proportion of hub nodes. It is important to distinguish vOT from the ventral anterior temporal region that has been implicated in multimodal conceptual representations specifically (Visser \& Lambon Ralph, 2011; Visser et al., 2012). Neuroanatomically, an important structural basis for this community may be provided by the inferior longitudinal fascicle, which runs from IPS over ventral occipital cortex and also connects to medial temporal cortex (Schmahmann \& Pandya, 2006).

Complementary to the analysis of the community structure our approach also allowed us to define the connections between the perisylvian and the ventral occipitotemporal subsystem. The two subsystems were connected through a unique link between left vOT and the posterior third of left GTm. This indicates the pivotal role of both vOT and GTm at the interface between these two communities. vOT was one of the regions with highest betweenness centrality, similarly to GTm and angular gyrus, confirming its pivotal role in the network. According to neurosurgical dissection of postmortem brains, these two structures are joined through the superficial layer of the inferior fronto-occipital fascicle, which connects superior parietal and basal temporal cortex with middle temporal gyrus and the inferior frontal gyrus (Martino et al., 2010; Sarubbo et al., 2011).

Apart from the perisylvian and the ventral occipitotemporal subsystem, the community structure revealed 4 additional communities (Fig. 3). The only node with high betweenness centrality lying outside the perisylvain or ventral occipitotemporal communities was the left inferior frontal junction. Left IFJ was part of a prefrontal subsystem with multiple nodes in inferior frontal sulcus, middle and superior frontal gyrus, both at the lateral convexity and to the medial side. It is plausible to attribute to this subsystem (Fig. 3, blue dots) a role in cognitive control (Derrfuss et al., 2005). Several of these nodes have been identified as hubs in previous studies (Buckner et al., 2009). 
To conclude, the temporal and occipital constituents of the 'associative-semantic network', defined by contrasting an explicit associative-semantic task with a visuoperceptual task with words and pictures, can be decomposed into two subsystems: a lateral temporal pathway that runs along the posterior third of the temporal gyrus and the superior temporal sulcus and is connected with inferior frontal areas and the angular gyrus, and, secondly, a lateral and ventral occipital pathway that runs anteriorly towards medial temporal cortex and is connected dorsally with the intraparietal sulcus. The transition zone between ventral occipital and temporal cortex, region vOT, belonged to the latter subsystem. These two systems are uniquely connected with each other through a link between GTm and vOT. Together, these two regions constitute the interface between these two communities.

\section{Acknowledgements}

This work was supported by Research Foundation Flanders (FWO) [G.0076.02, G0668.07 to R.V., G.0660.09 to R.V. and P.D.], K.U.Leuven [OT/08/056 to R.V. and M.V. PF/05/014 to R.V. and P.D.], Federaal Wetenschapsbeleid belspo [IUAP P6/29] and Stichting voor Alzheimer Onderzoek (SAO11020). N.N. is a postdoctoral fellow of the FWO and R.V. a senior clinical investigator of the FWO.

\section{References}

Batagelj, V., \& A.Mrvar (1998). Pajek: A program for large network analysis. Connections, 21, 47-57.

Binder, J. R., Desai, R. H., Graves, W. W., \& Conant, L. L. (2009). Where is the semantic system? a critical review and meta-analysis of 120 functional neuroimaging studies. Cereb Cortex, 19, 2767-2796.

Buckner, R., Koutstaal, W., Shacter, D., \& Rosen, B. (2000). Functional MRI evidence for a role of frontal and inferior temporal cortex in amodal components of priming. Brain, 123, 620-640.

Buckner, R. L., Sepulcre, J., Talukdar, T., Krienen, F. M., Liu, H., Hedden, T., AndrewsHanna, J. R., Sperling, R. A., \& Johnson, K. A. (2009). Cortical hubs revealed by intrinsic functional connectivity: mapping, assessment of stability, and relation to alzheimer's disease. J Neurosci, 29, 1860-1873.

Bullmore, E., \& Sporns, O. (2009). Complex brain networks: graph theoretical analysis of structural and functional systems. Nat Rev Neurosci, 10, 186-198.

Bullmore, E. T., \& Bassett, D. S. (2011). Brain graphs: graphical models of the human brain connectome. Annu Rev Clin Psychol, 7, 113-140.

Bundesen, C., \& Habekost, T. (2008). Principles of visual attention. Linking mind and brain. Oxford, UK: Oxford University Press.

Bundesen, C., Habekost, T., \& Kyllingsbaek, S. (2005). A neural theory of visual attention: Bridging cognition and neurophysiology. Psychol. Rev., 112, 291-328. 
Caspers, S., Eickhoff, S. B., Geyer, S., Scheperjans, F., Mohlberg, H., Zilles, K., \& Amunts, K. (2008). The human inferior parietal lobule in stereotaxic space. Brain Struct Funct, 212, 481-495.

Caspers, S., Geyer, S., Schleicher, A., Mohlberg, H., Amunts, K., \& Zilles, K. (2006). The human inferior parietal cortex: cytoarchitectonic parcellation and interindividual variability. Neuroimage, 33, 430-448.

Chertkow, H., Bub, D., Deaudon, C., \& Whitehead, V. (1997). On the status of object concepts in aphasia. Brain Lang., 58, 203-232.

Cohen, L., Dehaene, S., Naccache, L., Lehericy, S., Dehaene-Lambertz, G., Henaff, M., \& Michel, F. (2000). The visual word form area: Spatial and temporal characterization of an initial stage of reading in normal subjects and posterior split brain patients. Brain, 123, 291-307.

Cohen, L., Lehericy, S., Chochon, F., Lemer, C., Rivaud, S., \& Dehaene, S. (2002). Language-specific tuning of visual cortex? functional properties of the visual word form area. Brain, 125, 1054-1069.

Corbett, F., Jefferies, E., Ehsan, S., \& Lambon Ralph, M. A. (2009). Different impairments of semantic cognition in semantic dementia and semantic aphasia: evidence from the non-verbal domain. Brain, 132, 2593-2608.

Corbett, F., Jefferies, E., \& Ralph, M. A. L. (2011). Deregulated semantic cognition follows prefrontal and temporo-parietal damage: evidence from the impact of task constraint on nonverbal object use. J Cogn Neurosci, 23, 1125-1135.

Cristescu, T. C., Devlin, J. T., \& Nobre, A. C. (2006). Orienting attention to semantic categories. Neuroimage, 33, 1178-1187.

Demb, J., Desmond, J., Wagner, A., Vaidya, C., Glover, G., \& Gabrieli, J. (1995). Semantic encoding and retrieval in the left inferior prefrontal cortex: a functional MRI study of task difficulty and process specificity. J. Neurosci., 15, 5870-5878.

Derrfuss, J., Brass, M., Neumann, J., \& von Cramon, D. (2005). Involvement of the inferior frontal junction in cognitive control: Meta-analyses of switching and Stroop studies. Hum Brain Mapp, 25, 22-34.

Dmonet, J.-F., Thierry, G., \& Cardebat, D. (2005). Renewal of the neurophysiology of language: functional neuroimaging. Physiol Rev, 85, 49-95.

Goldberg, R., Perfetti, C., Fiez, J., \& Schneider, W. (2007). Selective retrieval of abstract semantic knowledge in left prefrontal cortex. J. Neurosci., 27, 3790-3798.

Gong, G., Rosa-Neto, P., Carbonell, F., Chen, Z. J., He, Y., \& Evans, A. C. (2009). Age- and gender-related differences in the cortical anatomical network. J Neurosci, 29, 15684-15693.

Griswold, M., Jakob, P., Heidemann, R., Nittka, M., Jellus, V., Wang, J., Kiefer, B., \& Haase, A. (2002). Generalized autocalibrating partially parallel acquisitions (GRAPPA). Magn Reson Med, 47, 1202-1210.

He, B. J., Shulman, G. L., Snyder, A. Z., \& Corbetta, M. (2007). The role of impaired neuronal communication in neurological disorders. Curr Opin Neurol, 20, 655-660. 
He, Y., Chen, Z., \& Evans, A. (2008). Structural insights into aberrant topological patterns of large-scale cortical networks in alzheimer's disease. J Neurosci, 28, 47564766.

He, Y., \& Evans, A. (2010). Graph theoretical modeling of brain connectivity. Curr Opin Neurol, 23, 341-350.

Heitger, M., Ronsse, R., Dhollander, T., Dupont, P., Caeyenberghs, K., \& Swinnen, S. (2012). Motor learning-induced changes in functional brain connectivity as revealed by means of graph theoretical network analysis. Neuroimage, in press.

Hellyer, P. J., Woodhead, Z. V. J., Leech, R., \& Wise, R. J. S. (2011). An investigation of twenty/20 vision in reading. $J$ Neurosci, 31, 14631-14638.

Hodges, J., Patterson, K., Oxbury, S., \& Funnell, E. (1992). Semantic dementia: Progressive fluent aphasia with temporal lobe atrophy. Brain, 115, 1783-1806.

Jefferies, E., \& Lambon Ralph, M. A. (2006). Semantic impairment in stroke aphasia versus semantic dementia: a case-series comparison. Brain, 129, 2132-2147.

Jobard, G., Crivello, F., \& Tzourio-Mazoyer, N. (2003). Evaluation of the dual route theory of reading: a metanalysis of 35 neuroimaging studies. Neuroimage, 20, 693-712.

Kamada, T., \& Kawai, S. (1989). An algorithm for drawing general undirected graphs. Information processing letters, 31, 7-15.

Lambon Ralph, M. A., Sage, K., Jones, R. W., \& Mayberry, E. J. (2010). Coherent concepts are computed in the anterior temporal lobes. Proc Natl Acad Sci U S A, 107, $2717-2722$.

Lauro-Grotto, R., Piccini, C., \& Shallice, T. (1997). Modality-specific operations in semantic dementia. Cortex, 33, 593-622.

Marrelec, G., Kim, J., Doyon, J., \& Horwitz, B. (2009). Large-scale neural model validation of partial correlation analysis for effective connectivity investigation in functional mri. Hum Brain Mapp, 30, 941-950.

Marrelec, G., Krainik, A., Duffau, H., Plgrini-Issac, M., Lehricy, S., Doyon, J., \& Benali, H. (2006). Partial correlation for functional brain interactivity investigation in functional mri. Neuroimage, 32, 228-237.

Martino, J., Brogna, C., Robles, S. G., Vergani, F., \& Duffau, H. (2010). Anatomic dissection of the inferior fronto-occipital fasciculus revisited in the lights of brain stimulation data. Cortex, 46, 691-699.

Mesulam, M. (1998). From sensation to cognition. Brain, 121, 1013-1052.

Mesulam, M. (2008). Representation, inference, and transcendent encoding in neurocognitive networks of the human brain. Ann Neurol, 64, 367-378.

Molenberghs, P., Gillebert, C., Peeters, R., \& Vandenberghe, R. (2008). Convergence between lesion-symptom mapping and fmri of spatially selective attention in the intact brain. J Neurosci, 28, 3359-3373. 
Nelissen, N., Dupont, P., Vandenbulcke, M., Tousseyn, T., Peeters, R., \& Vandenberghe, R. (2011). Right hemisphere recruitment during language processing in frontotemporal lobar degeneration and alzheimer's disease. J Mol Neurosci, 45, 637-647.

Nelissen, N., Laere, K. V., Thurfjell, L., Owenius, R., Vandenbulcke, M., Koole, M., Bormans, G., Brooks, D., \& Vandenberghe, R. (2009). Phase I study of the PIB derivative ${ }^{18} \mathrm{~F}$-flutemetamol in healthy volunteers and patients with probable Alzheimer's disease. J Nucl Med, 50, 1251-1259.

Nelissen, N., Pazzaglia, M., Vandenbulcke, M., Sunaert, S., Fannes, K., Dupont, P., Aglioti, S., \& Vandenberghe, R. (2010). Gesture discrimination in primary progressive aphasia: the intersection between gesture and language processing pathways. $J$ Neurosci, 30, 6334-6341.

Nelissen, N., Vandenbulcke, M., Fannes, K., Verbruggen, A., Peeters, R., Dupont, P., Laere, K. V., Bormans, G., \& Vandenberghe, R. (2007). A $\beta$ amyloid deposition in the language system and how the brain responds. Brain, 130, 2055-2069.

Nelson, S. M., Cohen, A. L., Power, J. D., Wig, G. S., Miezin, F. M., Wheeler, M. E., Velanova, K., Donaldson, D. I., Phillips, J. S., Schlaggar, B. L., \& Petersen, S. E. (2010). A parcellation scheme for human left lateral parietal cortex. Neuron, 67, 156-170.

Newman, M. E. J. (2006a). Finding community structure in networks using the eigenvectors of matrices. Phys Rev E Stat Nonlin Soft Matter Phys, 74, 036104.

Newman, M. E. J. (2006b). Modularity and community structure in networks. Proc Natl Acad Sci U S A, 103, 8577-8582.

Noonan, K. A., Jefferies, E., Corbett, F., \& Lambon Ralph, M. A. (2010). Elucidating the nature of deregulated semantic cognition in semantic aphasia: evidence for the roles of prefrontal and temporo-parietal cortices. J Cogn Neurosci, 22, 1597-1613.

Penny, W., Stephan, K., Mechelli, A., \& Friston, K. (2004). Comparing dynamic causal models. NeuroImage, 22, 1157-1172.

Price, C., \& Devlin, J. (2003). The myth of the visual word form area. Neuroimage, 19, $473-481$.

Price, C. J., \& Devlin, J. T. (2011). The interactive account of ventral occipitotemporal contributions to reading. Trends Cogn Sci, 15, 246-253.

Rogers, T., \& McClelland, J. (2004). Semantic cognition. A parallel distributed approach. Cambridge, MA, Massachusetts Institute of Technology Press.

Rubinov, M., \& Sporns, O. (2010). Complex network measures of brain connectivity: uses and interpretations. Neuroimage, 52, 1059-1069.

Sarubbo, S., De Benedictis, A., Maldonado, I. L., Basso, G., \& Duffau, H. (2011). Frontal terminations for the inferior fronto-occipital fascicle: anatomical dissection, dti study and functional considerations on a multi-component bundle. Brain Struct Funct, .

Schmahmann, J., \& Pandya, D. (2006). Fiber pathways of the brain. Oxford University Press. 
Seghier, M. L., Friston, K. J., \& Price, C. J. (2007). Detecting subject-specific activations using fuzzy clustering. Neuroimage, 36, 594-605.

Seghier, M. L., \& Price, C. J. (2011). Explaining left lateralization for words in the ventral occipitotemporal cortex. J Neurosci, 31, 14745-14753.

Sporns, O., Honey, C. J., \& Ktter, R. (2007). Identification and classification of hubs in brain networks. PLoS One, 2, e1049.

Thierry, G., \& Price, C. (2006). Dissociating verbal and nonverbal conceptual processing in the human brain. J. Cogn. Neurosci., 18, 1018-1028.

van den Heuvel, M. P., Mandl, R. C. W., Stam, C. J., Kahn, R. S., \& Hulshoff Pol, H. E. (2010). Aberrant frontal and temporal complex network structure in schizophrenia: a graph theoretical analysis. J Neurosci, 30, 15915-15926.

Van Doren, L., Dupont, P., DeGrauwe, S., Peeters, R., \& Vandenberghe, R. (2010). The amodal system for conscious word and picture identification in the absence of a semantic task. Neuroimage, 49, 3295-3307.

Van Doren, L., Schrooten, M., Adamczuk, K., Dupont, P., \& Vandenberghe, R. (2012). Chronometry of conscious word and picture identification: common and modalityspecific effects. Neuroimage, in press.

Vandenberghe, R., Geeraerts, S., Molenberghs, P., Lafosse, C., Vandenbulcke, M., Peeters, K., Peeters, R., Hecke, P. V., \& Orban, G. (2005). Attentional responses to unattended stimuli in human parietal cortex. Brain, 128, 2843-2857.

Vandenberghe, R., \& Gillebert, C. (2009). Parcellation of human parietal cortex: converging evidence from functional imaging in the intact human brain and patient lesion studies (invited review). Behav Brain Res, 199, 171-182.

Vandenberghe, R., Price, C., Wise, R., Josephs, O., \& Frackowiak, R. (1996). Functional anatomy of a common semantic system for words and pictures. Nature, 383, 254-256.

Vandenbulcke, M., Peeters, R., Dupont, P., Van Hecke, P., \& Vandenberghe, R. (2007). Word reading and posterior temporal dysfunction in amnestic mild cognitive impairment. Cereb. Cortex, 17, 542-551.

Vandenbulcke, M., Peeters, R., Fannes, K., \& Vandenberghe, R. (2006). Knowledge of visual attributes in the right hemisphere. Nat. Neurosci., 9, 964-970.

Vandenbulcke, M., Peeters, R., Hecke, P. V., \& Vandenberghe, R. (2005). Anterior temporal laterality in primary progressive aphasia shifts to the right. Ann. Neurol., 58, $362-370$.

Vinckier, F., Dehaene, S., Jobert, A., Dubus, J., Sigman, M., \& Cohen, L. (2007). Hierarchical coding of letter strings in the ventral stream: dissecting the inner organization of the visual word-form system. Neuron, 55, 143-156.

Visser, M., Embleton, K. V., Jefferies, E., Parker, G. J., \& Ralph, M. A. L. (2010). The inferior, anterior temporal lobes and semantic memory clarified: novel evidence from distortion-corrected fmri. Neuropsychologia, 48, 1689-1696. 
Visser, M., Jefferies, E., Embleton, K. V., \& Lambon Ralph, M. A. (2012). Both the middle temporal gyrus and the ventral anterior temporal area are crucial for multimodal semantic processing: Distortion-corrected fmri evidence for a double gradient of information convergence in the temporal lobes. J Cogn Neurosci, 24, 1766-1778.

Visser, M., \& Lambon Ralph, M. A. (2011). Differential contributions of bilateral ventral anterior temporal lobe and left anterior superior temporal gyrus to semantic processes. J Cogn Neurosci, 23, 3121-3131.

Wagner, A., Desmond, J., Demb, J., Glover, G., \& Gabrieli, J. (1997). Semantic repetition priming for verbal and pictorial knowledge: a functional MRI study of left inferior prefrontal cortex. J. Cogn. Neurosci., 9, 714-726.

Whitney, C., Kirk, M., O’Sullivan, J., Lambon Ralph, M. A., \& Jefferies, E. (2011). The neural organization of semantic control: Tms evidence for a distributed network in left inferior frontal and posterior middle temporal gyrus. Cereb Cortex, 21, 1066-1075.

Whitney, C., Kirk, M., O'Sullivan, J., Lambon Ralph, M. A., \& Jefferies, E. (2012). Executive semantic processing is underpinned by a large-scale neural network: revealing the contribution of left prefrontal, posterior temporal, and parietal cortex to controlled retrieval and selection using tms. J Cogn Neurosci, 24, 133-147.

Wise, R., Scott, S., Blank, S., Mummery, C., Murphy, K., \& Warburton, E. (2001). Separate neural subsystems within "Wernicke's area". Brain, 124, 83-95.

Woodhead, Z. V. J., Brownsett, S. L. E., Dhanjal, N. S., Beckmann, C., \& Wise, R. J. S. (2011). The visual word form system in context. J Neurosci, 31, 193-199. 